\title{
Acute disseminated encephalomyelitis presenting as multiple cystic lesions
}

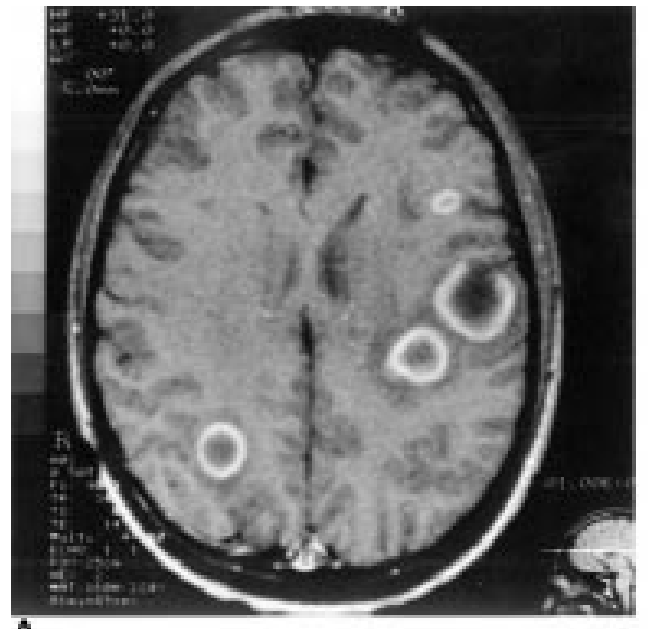

A

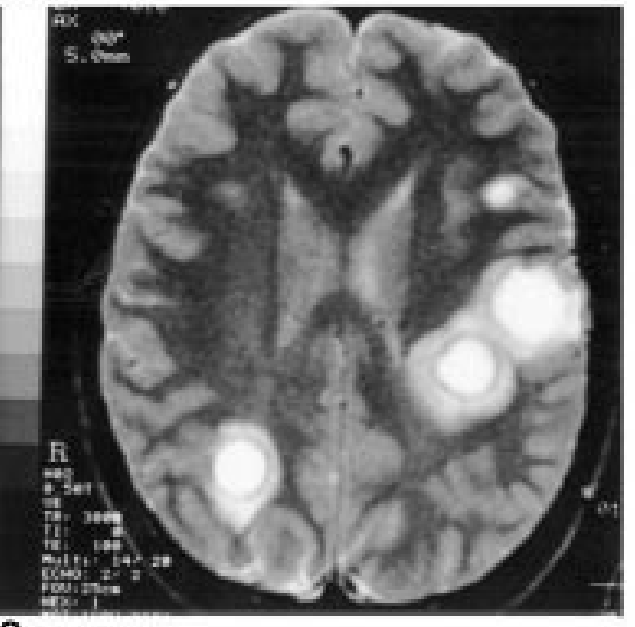

C

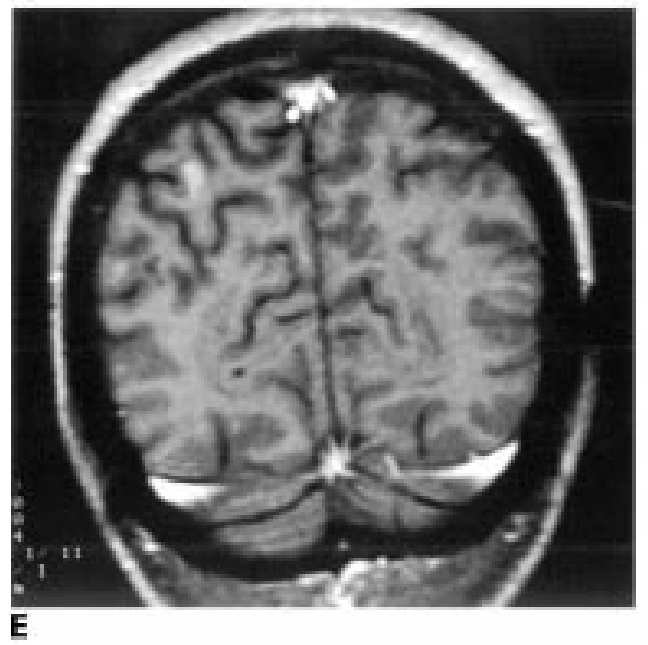

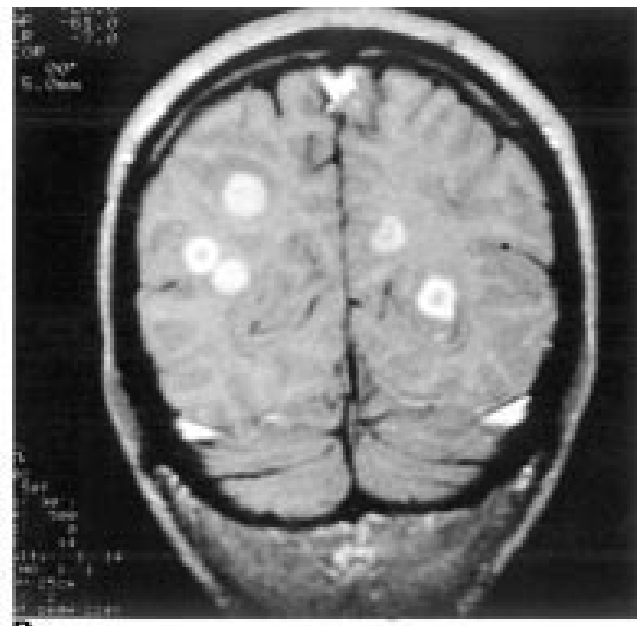

$\bar{B}$

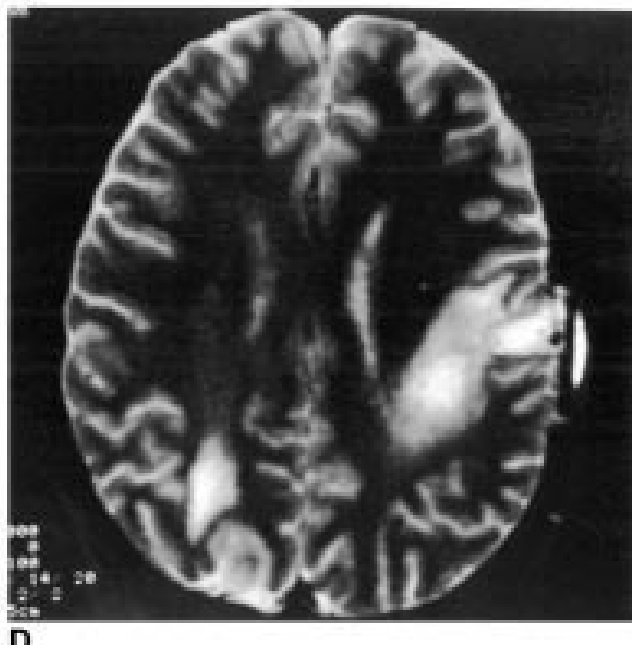

A 23 year old right handed man presented with a left parietal syndrome two weeks after a non-specific upper respiratory tract infection. Other clinical examination was normal. Cranial MRI disclosed multiple supratentorial lesions predominating in white matter. $\mathrm{T} 1$ weighted images with Ga-DTPA contrast disclosed ring shaped enhancing hypointense lesions (figure, A, B). In axial T2 weighted images these lesions were hyperintense with slight surrounding oedema (figure, C). Antibody tests for HIV, cysticercosis, and toxoplasma were negative. Thoracic and abdominal CT was normal. Because of rapid clinical deterioration, steroid treatment was begun and surgical biopsy carried out. Histology showed a subacute myelonoclastic process with many macrophages and relative sparing of axons. Three weeks after admission, MRI showed regression of the T2 lesions (figure, D) with almost total disappearance of T1 contrast enhancement and hypointensities (figure, E) No additional lesions were seen one month and one year later and there was total disappearance of contrast enhancement and supplementary regression of hyperintense lesions. Clinically he had improved. One year after onset, he had a mild memory disorder, episodic dysarthria, and some sensory loss in his right hand.

A DE RECONDO Department of Neurology J P GUICHARD

Department of Neuroradiology, Lariboisière Hospital, Paris, France Correspondence to: Dr A de Recondo, Department of Neurology, Lariboisière Hospital, 2 rue Ambroise Paré 75475 Paris 10 ème, France. 